Pirineos.Revista de Ecología de Montaña Vol. 165, 193-213

Jaca, Enero-Diciembre, 2010

ISSN: 0373-2568

eISSN: 1988-428

doi: 10.3989/Pirineos.2010.165010

\title{
LA VARIABILIDAD TEMPORAL DE LA RESPUESTA HIDROLÓGICA EN UNA PEQUEÑA CUENCA MEDITERRÁNEA FORESTADA DEL PIRINEO CENTRAL
}

\author{
P. SERrano-Muela ${ }^{1}$, D. ReGÜÉS ${ }^{1} \&$ E. NADAl-ROMERO ${ }^{1,2}$ \\ 1 Instituto Pirenaico de Ecología (CSIC), Campus de Aula Dei, Avenida Montañana 1005, 50192, \\ Zaragoza, Spain. \\ 2 Dep. Earth and Environmental Sciences, Physical and Regional Geography Research Group, \\ K.U.Leuven. Celestijnenlaan 200 E, P. O. box 3001.Heverlee, Belgium. \\ e-mail: pili@ipe.csic.es
}

ABSTRACT.- This paper analyses the stormflow response of the San Salvador forested catchment $\left(0.92 \mathrm{~km}^{2}\right)$ located in the Central Pyrenees, Spain. Using stormflow coefficient and discharge increment, the temporal variability of the rainfallrunoff relationship is analyzed at the event scale from hydrographs observed over a 7-year period. Results show that the storm-flow coefficient has a clear seasonal pattern with an alternance between a wet period, when the catchment is hydrologically responsive, and a dry summer period, when the catchment presents almost no reaction to any rainfall. The relationships of the storm-flow coefficient and discharge increment with several hydrological variables shows similar (but lower) correlations than usually observed in other Mediterranean or temperate catchments and demonstrates that the storm flow response is mainly depending on water table with rainfall depth and base flow as secondary factors. The marked seasonality observed in the San Salvador catchment is most likely accentuated by forest that cover all the catchment, but this deserves further investigations.

Keywords: Forest hydrology; baseflow, stormflow response; water table; SubMediterranean catchment.

RESUMEN.-El análisis de los procesos hidrológicos en zonas forestales es objeto de numerosos estudios, debido a los cambios ambientales que se están produciendo en los últimos años y a los cambios experimentados en los usos del suelo desde las últimas décadas, como resultado del proceso de revegetación. Este trabajo analiza la respuesta hidrológica de la pequeña cuenca forestal de San Salvador $\left(0.92 \mathrm{~km}^{2}\right)$, localizada en el Pirineo Central, España. Se ha analizado la variabilidad de las relaciones precipitación-caudal a escala de evento, durante un periodo de estudio de 7 años, 
teniendo en cuenta los coeficientes de escorrentía de crecida y el incremento de la descarga. Los resultados muestran que el coeficiente de escorrentía presenta un gran patrón estacional con una alternancia entre periodos húmedos, cuando la cuenca tiene capacidad de responder hidrológicamente, y periodos secos, cuando la cuenca casi no reacciona a ninguna precipitación. La relación entre el coeficiente de escorrentía y el incremento de la descarga con otras variables hidrológicas muestra correlaciones similares (aunque siempre más bajas) que las observadas en otras cuencas mediterráneas y templadas, y demuestra que la escorrentía depende principalmente de la profundidad de la capa freática y del flujo de base como factor secundario. La marcada estacionalidad observada en la cuenca de San Salvador es probablemente más acentuada debido a la densa cubierta forestal, pero este hecho merece futuras investigaciones.

Palabras clave: Hidrología forestal; flujo de base; respuesta hidrológica; capa freática; cuenca submediterránea.

\section{Introducción}

A pesar de la controversia existente sobre el papel del bosque (véanse, por ejemplo, las síntesis de BOSCH \& HEWLETT, 1982; WHITEHEAD \& ROBINSON, 2003; ANDRÉASSIAN, 2004), los estudios en cuencas experimentales (LATRON, 2003; GUOJING et al., 2005; COSANDEY et al., 2005; LANA-RENAULT, 2007; GARCÍA-RUIZ et al., 2008; NADALROMERO, 2008) han proporcionado datos de gran valor para evaluar de manera objetiva el efecto del tipo de cubierta vegetal sobre la respuesta hidrológica y, en particular sobre la generación de crecidas.

La percepción clásica de que el bosque incrementa los recursos hídricos ha sido un tema seriamente cuestionado por los resultados científicos obtenidos en la segunda mitad del siglo XX, ya que la presencia de una cobertura de bosque supone, como así se ha demostrado, una reducción de la cantidad de agua disponible para la escorrentía (HIBBERT, 1967; LULL \& REINHART 1967; BOSCH \& HEWLETT, 1982; TRIMBLE, 1987; STEDNICK, 1996). Más recientemente ANDRÉASSIAN (2004) y COSANDEY et al. (2005) pusieron de manifiesto que el contraste entre los suelos desnudos y los vegetados es lo que mejor explica las diferencias en la respuesta hidrológica a escala de cuenca.

Actualmente, el análisis de los procesos hidrológicos en zonas forestales es objeto de numerosos estudios (COSANDEY et al., 2005; MARTÍNEZFERNÁNDEZ et al., 2005; ZABALETA, 2007; SERRANO-MUELA et al., 2008b; LATRON \& GALLART, 2008; LANA-RENAULT et al., 2009) debido fundamentalmente a los cambios ambientales que se están produciendo en 
los últimos años, así como a los cambios que han experimentado los usos del suelo desde las últimas décadas. Este hecho está siendo de gran trascendencia en las regiones del ámbito mediterráneo, a cuyo contexto pertenece este trabajo, donde se ha observado una tendencia negativa en los caudales que coincide con el progresivo incremento de las áreas forestales (BEGUERÍA et al., 2003; LÓPEZ-MORENO et al., 2008).

El presente trabajo tiene por objetivo profundizar en el estudio de la respuesta hidrológica en la cuenca de San Salvador y mejorar la comprensión sobre los distintos procesos que intervienen en la generación de crecidas en un ámbito forestal. Para ello se han analizado 7 años de registros de precipitación y caudal, teniendo en cuenta distintas escalas temporales y la variabilidad estacional. Sin embargo, también se ha tomado en consideración la información adquirida mediante estudios específicos sobre procesos que intervienen en la generación de escorrentía (interceptación y dinámica del nivel freático).

\section{2. Área de estudio}

La cuenca de San Salvador $\left(0,92 \mathrm{~km}^{2}\right)$ se localiza en el valle de Aísa, en el sector del Flysch Eoceno surpirenaico. Más del $90 \%$ de su superficie presenta una densa cubierta forestal (excepto un pequeño rodal con bosque abierto y matorral que fue cultivado hasta comienzos del siglo XX), constituyendo un ejemplo de ambiente natural en montaña media (Figura 1).

La cuenca de San Salvador es de gran interés por tratarse de un ambiente que ha sufrido poco el efecto de la agricultura y la ganadería, mientras un elevado porcentaje de laderas pirenaicas están afectadas por fuertes modificaciones, tales como aterrazamiento, deforestación y eliminación de la vegetación original.

Las laderas están cubiertas por un coluvión pedregoso. La homogeneidad litológica explica la ausencia de grandes contrastes en la forma y pendiente de las laderas. La parte baja de la cuenca está a $830 \mathrm{~m}$, y la divisoria a $1295 \mathrm{~m}$. La pendiente media es del $26 \%$.

Las definiciones del clima de la zona de estudio han sido numerosas y siempre como de transición entre características de clima húmedo (de tipo atlántico) y características mediterráneas, con cierto carácter continental (PUIGDEFÁBREGAS \& CREUS, 1976; CREUS, 1983). La temperatura media anual oscila entre 9 y $10^{\circ} \mathrm{C}$. La precipitación media anual en la localidad de Aísa, a $6 \mathrm{~km}$ hacia el noreste, es de $1100 \mathrm{~mm}$ y se reparte principalmente entre los equinoccios. En verano se registra un elevado déficit pluviométrico. 


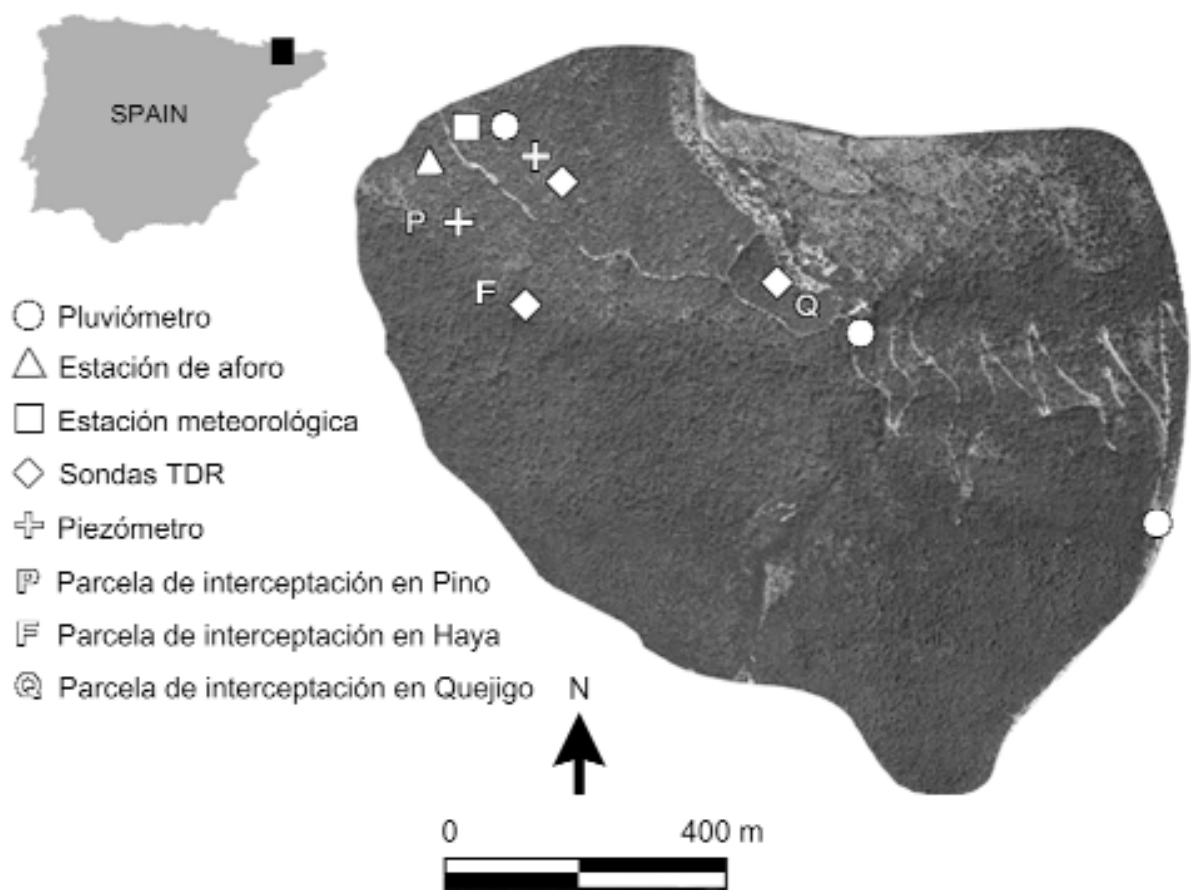

Figura 1. Vista aérea de la cuenca de San Salvador y localización de los principales instrumentos de medida.

Figure 1. Aerial view of the San Salvador catchment and location of the main instruments.

La mayor parte de la superficie de la cuenca está cubierta por un denso bosque. En la vertiente umbría domina la presencia de pino silvestre (Pinus sylvestris), mezclado con bosquetes de haya (Fagus sylvatica) en las zonas más húmedas. El sotobosque es denso y está formado mayoritariamente por boj (Buxus sempervirens). En solana el pino silvestre se mezcla con el quejigo (Quercus faginea) (MONTSERRAT, 1971). El sotobosque es predominantemente de boj pero aparece mezclado con otras especies espinosas mediterráneas de porte bajo, como la aliaga (Genista scorpius), el enebro (Juniperus communis) y el erizón (Echinospartum horridum), ésta última en las cotas superiores.

Los suelos de la cuenca son de tipo forestal. De forma general presentan un buen desarrollo y estructura, sobre todo en la vertiente norte, donde se encuentran más frecuentemente suelos de tipo Kastanozem o Phaeozem. Son muy ricos en materia orgánica, pues el aporte de hojarasca es continuo y 
abundante. Los suelos de la vertiente solana están algo menos desarrollados y presentan mayor heterogeneidad, mostrando menor desarrollo en las cotas más altas de la cuenca, donde las pendientes son más elevadas. Predominan en esta ladera los suelos de tipo Cambisol y Kastanozem, éstos últimos en condiciones más evolucionadas, pero también de tipo Regosol, donde la elevada pendiente impide un mayor desarrollo.

\section{Metodología}

La instrumentación para el estudio hidrológico de la cuenca de San Salvador fue instalada en el año 1999 (Figura 1). Consta de una estación de aforo (de tipo H-Flume). El nivel de agua se mide con un sensor de ultrasonidos y con una sonda de presión con sistema de almacenamiento de datos propio (Keller DCX-22 AA). Asimismo, la estación de aforo cuenta con muestreador automático (ISCO-3700) que se activa durante las crecidas y permite evaluar las concentraciones de sedimento en solución y en suspensión. Junto al aforador hay una estación meteorológica. Además hay tres pluviómetros de balancín (Davis instrument) para el registro de la lluvia, localizados según un gradiente altitudinal ( 880 m, 1080 m y 1270 m). También hay dos piezómetros para la medición en continuo de los niveles piezométricos, en vertiente solana y umbría, en la parte baja de la cuenca, así como tres parcelas para la medición de la interceptación de la lluvia y un conjunto de sondas TDR para la medición continua de la humedad.

El análisis de este trabajo se ha llevado a cabo a escala de crecida. Para individualizar los eventos se utilizó el criterio clásico de HEWLETT \& HIBBERT (1967) pero la recta cuyo valor era de 13,1 $1 \mathrm{~s}^{-1} \mathrm{~km}^{-2}$ día-1 ha sido ajustada a la morfología y las características de un hidrograma representativo como ya hicieran LATRON (2003), LANA-RENAULT, (2007) y NADALROMERO (2008), obteniéndose un valor sensiblemente menor, 1,83 $1 \mathrm{~s} \mathrm{sm}^{-1}$ día $^{-1}$, al citado anteriormente.

\section{Resultados}

El análisis se ha realizado para un periodo de 7 años hidrológicos, desde octubre de 1999 hasta septiembre de 2006. Se analizaron 91 eventos de precipitación (superiores a $8 \mathrm{~mm}$ ), independientemente de que tuvieran respuesta hidrológica -manifestada como incremento de caudal- o no.

La media de precipitación para el periodo estudiado es de $930 \mathrm{~mm}( \pm 252$ $\mathrm{mm}$ ), distribuidos con una marcada estacionalidad. La primavera y el otoño 
son los periodos más húmedos en la cuenca de San Salvador, concentrándose entre estos periodos más del $60 \%$ de la precipitación anual. El invierno tiende a ser bastante seco, aunque se registran algunas lluvias ligadas al paso de frentes. El verano es seco, aunque pueden darse tormentas ocasionales, a veces de gran magnitud (GARCÍA-RUIZ et al., 2000). La magnitud de los eventos registrados se distribuye de la siguiente manera: el $38 \%$ tiene un volumen menor de $20 \mathrm{~mm}$, un $32 \%$ tiene un volumen entre 20 y $40 \mathrm{~mm}$, y un $30 \%$ de los eventos de lluvia son mayores de $40 \mathrm{~mm}$.

La gran mayoría de los eventos estudiados se han producido sobre todo en las estaciones húmedas, estando el rango de las precipitaciones entre $8 \mathrm{y}$ $84 \mathrm{~mm}$ la más voluminosa en el periodo de estudio (mediana $=25 \mathrm{~mm}$ ). Los valores de pico de caudal oscilan entre $0,21 \mathrm{~s}^{-1}$ y $4701 \mathrm{~s}^{-1}$ La mayor parte de los eventos tiene un pico de caudal moderado, y sólo cuatro superan los 400 $1 \mathrm{~s}^{-1}$. La distribución estacional de los eventos analizados es la siguiente: 35 en otoño, 12 en invierno, 27 en primavera y 17 en verano.

En la Figura 2 se representa la evolución anual del coeficiente de escorrentía de crecida $\left(\mathrm{C}_{\mathrm{ec}}\right)$ para los eventos seleccionados. En este gráfico, se observa la clara estacionalidad del $\mathrm{C}_{\mathrm{ec}}$ durante el año. Todos los eventos cuyos $\mathrm{C}_{\mathrm{ec}}$ sobrepasa el 0,10 se producen entre otoño, primavera e invierno. En verano raramente se produce respuesta.

Se pueden distinguir tres periodos:

1) Un periodo húmedo entre noviembre y abril en el cual los $C_{e c}$ varían entre 0 y 0,50 . En este caso, la mayor parte de los volúmenes de escorrentía son superiores al 1\%, salvo cuando las precipitaciones son inferiores a $10 \mathrm{~mm}$.

2) Un periodo seco entre mediados de junio y mediados de septiembre, en el cual $\operatorname{los} \mathrm{C}_{\mathrm{ec}}$ son generalmente inferiores a 0,01 .

3) Un periodo de transición de condiciones húmedas a secas (entre mayo y junio) y de condiciones secas a húmedas (entre finales de septiembre y principios de octubre). En estos periodos es cuando se pueden encontrar los $\mathrm{C}_{\mathrm{ec}}$ más variables, en general inferiores a 0,10.

Se han obtenido los coeficientes de correlación lineal entre algunas de las variables características de las crecidas estudiadas con el objetivo de examinar la influencia de los diversos factores en la magnitud de la respuesta. Por un lado, el coeficiente de escorrentía de crecida $\left(\mathrm{C}_{\mathrm{ec}}\right)$ y el incremento máximo de caudal $(\Delta \mathrm{Q})$, $\mathrm{y}$, por otro, el volumen total de precipitación $(\mathrm{P})$, la intensidad máxima de lluvia $\left(\mathrm{I}_{\max }\right)$ y el caudal de base $\left(\mathrm{Q}_{\mathrm{b}}\right)$.

Los resultados (Tabla 1) muestran que la correlación entre $C_{\text {ec }}$ y P sólo es significativa cuando los eventos de precipitación son superiores a $20 \mathrm{~mm}$. La correlación entre $C_{e c} y_{Q_{b}}$ es siempre significativa, independientemente del volumen de la lluvia, pero para pequeñas lluvias $(<20 \mathrm{~mm})$ el $\mathrm{C}_{\mathrm{ec}}$ depende 
LA VARIABILIDAD TEMPORAL DE LA RESPUESTA HIDROLÓGICA EN UNA PEQUEÑA...

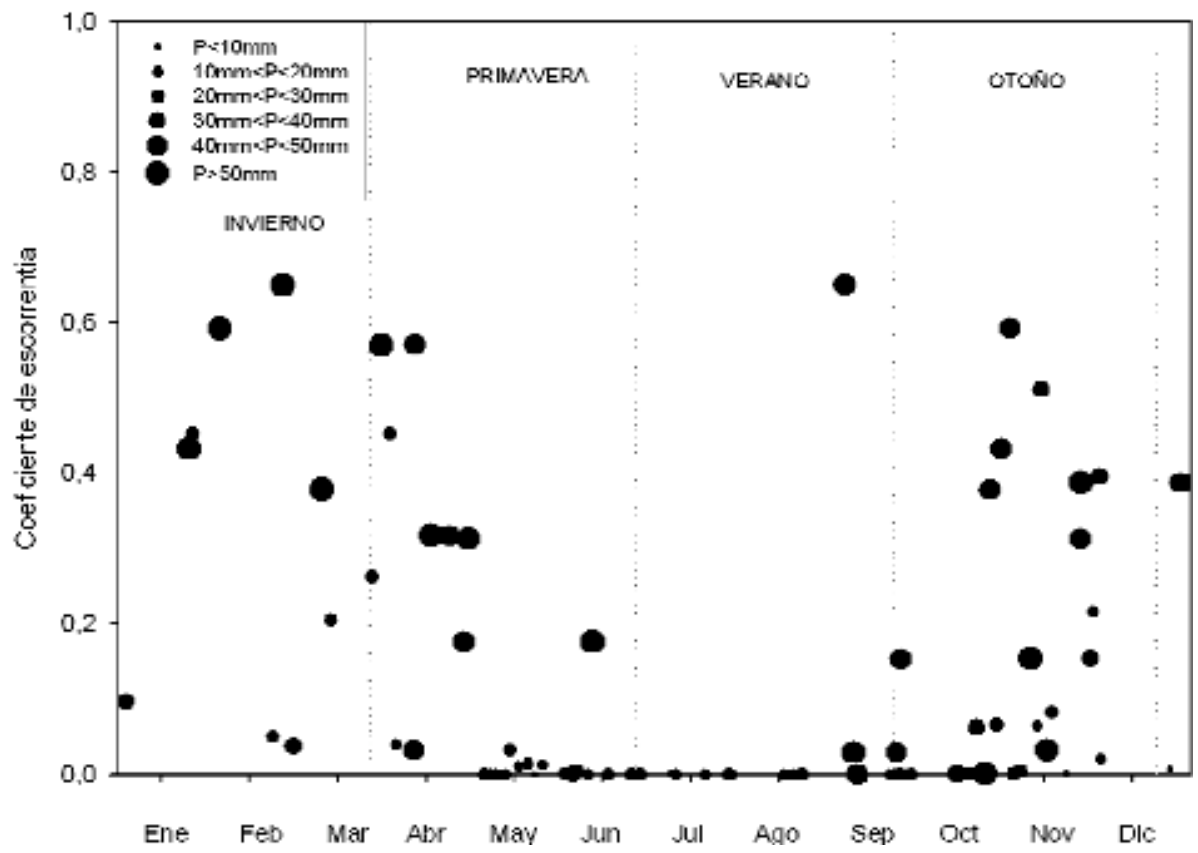

Figura 2. Evolución del coeficiente de escorrentía a lo largo del año en la cuenca de San Salvador. Figure 2. Seasonal evolution of the store-flow coefficient (CE) throughout the year.

Tabla 1. Coeficientes de correlación lineal del coeficiente de escorrentía de crecida y del incremento máximo de caudal con el volumen total de precipitación, la intensidad máxima de lluvia y el caudal de base. Las correlaciones con asterisco son estadísticamente significativas $(p<0.01)$. El total de eventos analizados fueron 91. Los eventos se separaron en dos grupos en función de la precipitación ( $\mathrm{P}<20 \mathrm{~mm} ; \mathrm{n}=35$ y con $\mathrm{P}>20 \mathrm{~mm} ; \mathrm{n}=56)$

Table 1. Lineal correlations of the storm-flow coefficient and the maximum increase of flow the total volume of rainfall, maximum rainfall intensity and baseflow. ${ }^{*}$ Correlations are significant at $p<0.01$. Total events analysed were 91. The events were separated in two groups depend on the rainfall $(P<20 \mathrm{~mm} ; n=35$ and with $P>20 m m ; n=56)$.

\begin{tabular}{|lcccc|}
\hline & \multicolumn{3}{c|}{$P<20 \mathrm{~mm}$} & \multicolumn{2}{c|}{$P>20 \mathrm{~mm}$} \\
\hline & $\begin{array}{c}C_{e c} \\
(\%)\end{array}$ & $\begin{array}{c}\Delta Q \\
\left(l s^{-1} \mathrm{~km}^{-2}\right)\end{array}$ & $\begin{array}{c}C_{e c} \\
(\%)\end{array}$ & $\begin{array}{c}\Delta Q \\
\left(l s^{-1} \mathrm{~km}^{-2}\right)\end{array}$ \\
\hline $\mathrm{P}(\mathrm{mm})$ & 0.24 & 0.26 & $0.46^{*}$ & $0.66^{*}$ \\
$\mathrm{I}_{\text {max }}\left(\mathrm{mmh}^{-1}\right)$ & -0.22 & -0.17 & -0.25 & -0.00 \\
$\mathrm{Q}_{\mathrm{b}}\left(\mathrm{ss}^{-1} \mathrm{~km}^{-2}\right)$ & $0.70^{*}$ & $0.68^{*}$ & $0.67^{*}$ & 0.32 \\
\hline
\end{tabular}


casi exclusivamente del $\mathrm{Q}_{\mathrm{b}^{\prime}}$ y cuando la lluvia es más voluminosa (>20 mm), en esa dependencia interviene también la precipitación total. La variación máxima de caudal $(\Delta \mathrm{Q})$ muestra correlaciones significativas exclusivamente con el $\mathrm{Q}_{\mathrm{b}^{\prime}}$ para eventos de precipitación inferiores a $20 \mathrm{~mm}$, y con P cuando el evento es superior a $20 \mathrm{~mm}$. Por otro lado, los resultados muestran que la intensidad máxima no tiene influencia ninguna sobre la magnitud de la respuesta hidrológica, pues no existe ninguna correlación significativa entre $I_{\max }$ y el resto de variables.

Con la finalidad de destacar las relaciones existentes entre la precipitación (mm), el caudal de base $\left(1 \mathrm{~s}^{-1} \mathrm{~km}^{-2}\right)$ y el coeficiente de escorrentía, se han relacionado todas estas variables en la Figura 3, siguiendo la propuesta de COSANDEY (1986).

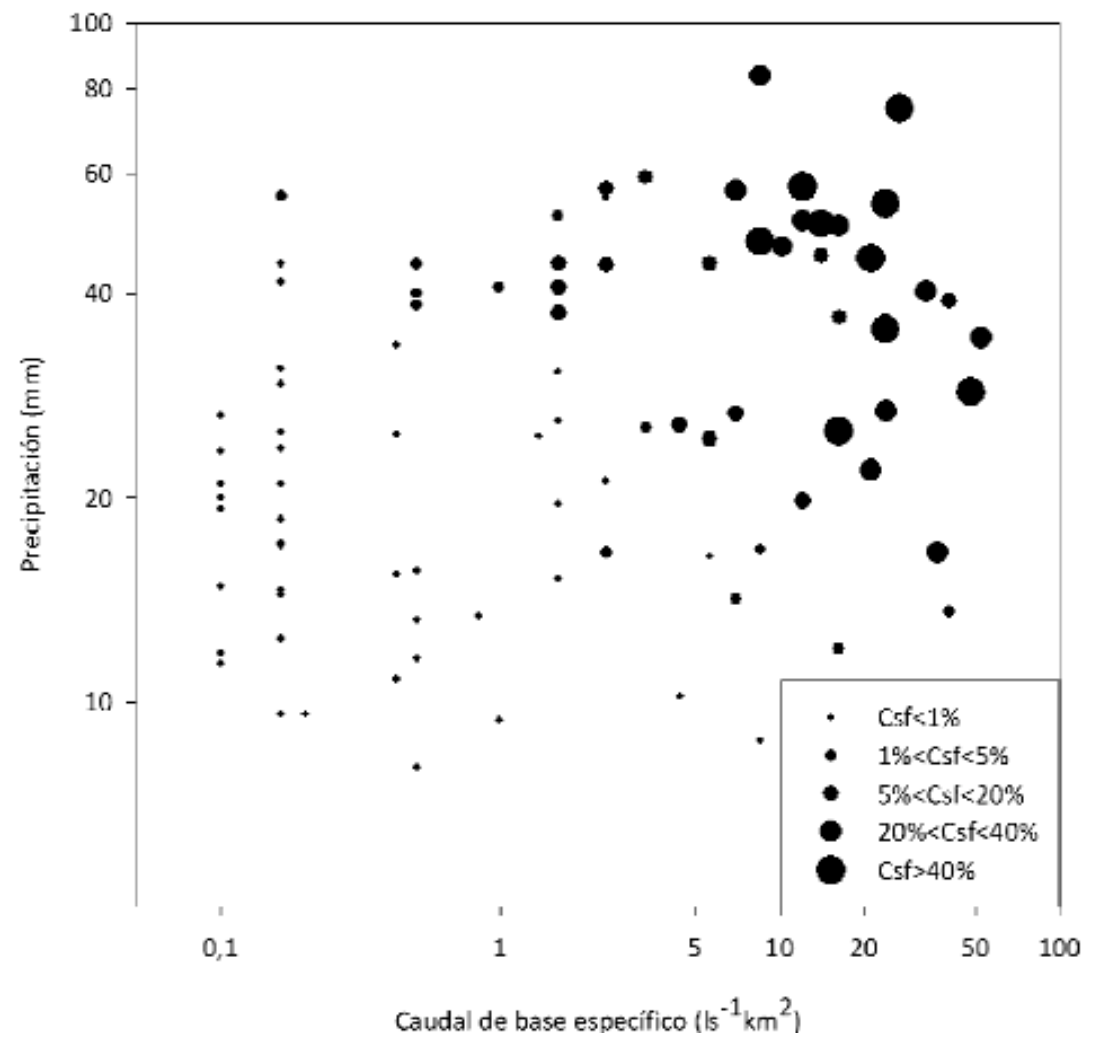

Figura 3. Coeficientes de escorrentía de crecida (Csf) observados en función del caudal de base y de la precipitación en la cuenca de San Salvador.

Figure 3. Store-flow coefficients (Csf) observed in relation to the basedflow and rainfall of the Araguas catchment. 
El rango de valores del $\mathrm{C}_{\mathrm{ec}}$ oscila entre 0 y el 0,65, situándose la mediana en el valor de 0,01 . Un $25 \%$ de los casos presenta un $\mathrm{C}_{\mathrm{ec}}$ superior a 0,15 (23 casos) Sin embargo, casi el 50\% de los casos (45) tiene coeficientes inferiores a 0,10 . Puede observarse que una lluvia considerada voluminosa, en torno a los $40 \mathrm{~mm}$, genera una respuesta diversa en función del caudal de base inmediatamente anterior a la crecida. Si el caudal de base es muy bajo (inferior de $1 \mathrm{l} \mathrm{s}^{-1} \mathrm{~km}^{-2}$ ), el coeficiente de escorrentía lo es también, no superando en ningún caso el 0,01, mientras que si el caudal de base presenta valores medios o altos (a partir de $20 \mathrm{l} \mathrm{s}^{-1} \mathrm{~km}^{-2}$ ) el coeficiente de escorrentía alcanza frecuentemente un valor elevado, pudiendo llegar hasta 0,50. De manera similar, para un caudal de base determinado, se observa que la respuesta hidrológica de la cuenca puede ser muy variable en función de la magnitud del evento de lluvia y que es necesario un volumen de lluvia elevado para obtener coeficientes de escorrentía altos. Cuando el caudal de base es moderado las precipitaciones de menos de $10 \mathrm{~mm}$ no causan nunca respuestas con coeficientes de escorrentía mayores de 0,01, mientras los eventos de menos de $20 \mathrm{~mm}$ producen también respuestas muy moderadas, en ningún caso los coeficientes de escorrentía superan el 0,05

En la Figura 4 se han representado los hidrogramas, hietogramas y niveles piezométricos registrados durante dos periodos (húmedo y seco) sucesivos. En el caso del nivel freático se muestran los datos correspondientes al piezómetro instalado en la vertiente solana, pues la serie temporal de datos es algo mayor que la de la vertiente umbría. Además la dinámica estacional es bastante similar en ambos casos. La información piezométrica se ha utilizado como indicador del estado hídrico de los suelos.

Tal como muestra la evolución del nivel freático, entre los meses de enero y abril de 2006 transcurre el periodo más húmedo con las respuestas hidrológicas más importantes del año. Destaca principalmente el hecho de que la magnitud de las crecidas está estrechamente relacionada con la altura del nivel freático. La precipitación acumulada en el periodo húmedo fue de $317,6 \mathrm{~mm}$ y la escorrentía total fue de $104,5 \mathrm{~mm}$ (con un coeficiente de escorrentía de 0,33 ). Se observan algunas diferencias interesantes entre la respuesta hidrológica al principio y al final de la estación húmeda.

$\mathrm{Al}$ principio del periodo húmedo las respuestas hidrológicas presentan bajos coeficientes de escorrentía, con caudales máximos de muy baja magnitud e hidrogramas de forma prácticamente plana, tal y como se puede apreciar en las crecidas registradas entre enero y principios de marzo (Figura 2). Esta respuesta tan baja se explica fundamentalmente por las características de las precipitaciones registradas durante el invierno, frecuentemente en forma de nieve y generalmente caracterizadas por mostrar intensidades muy bajas y volúmenes entre bajos y moderados. A pesar de ello, el nivel freático 

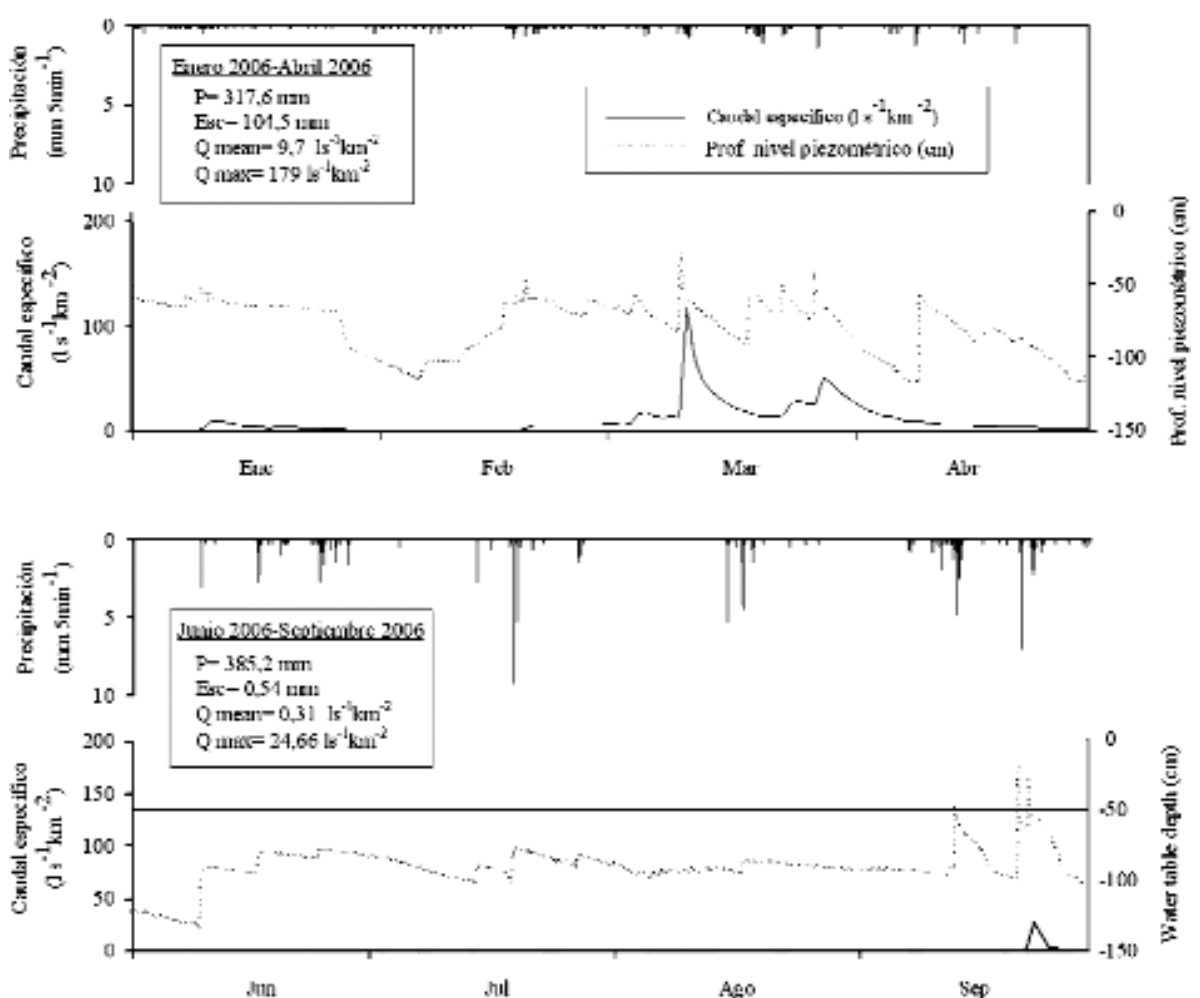

Figura 4. Relación entre precipitación y caudal durante dos periodos sucesivos (el primero entre enero y abril de 2006 y el segundo entre junio y septiembre de 2006. Se muestra la fuerte estacionalidad de la respuesta. La línea contínua muestra el límite del suelo a partir del cual se producen respuestas hidrológicas con aumento de caudal.

Figure 4. Relationship between rainfall and flow during two consecutive periods (the first one between January and April 2006 and second one between June and September 2006). The high response seasonality is shown. The solid line shows the limit of the soil from hydrological responses are produced with flow increases.

se mantiene muy elevado durante varias semanas consecutivas, como consecuencia de la escasísima evapotranspiración potencial que se produce en este momento del año. Asimismo, los valores medios de interceptación de la lluvia son más bajos que en otros momentos el año, alrededor del $20 \%$ (SERRANO-MUELA et al., 2008a) debido a que las especies caducifolias carecen de hojas en este periodo.

Hacia la segunda mitad del periodo húmedo, entre finales de invierno y principios de primavera, a medida que se incrementa la ocurrencia de las 
precipitaciones, la altura del nivel freático indica que la cuenca presenta un estado de humedad elevado, los coeficientes de escorrentía aumentan y alcanzan valores que incluso pueden superar el 0,50 (Figura 2). En consecuencia los picos máximos de caudal alcanzan los valores mayores de todo el año hidrológico. Además, los hidrogramas de crecida muestran rápidos tramos ascendentes de caudal y curvas de recesión mucho más lentas y prolongadas. Todo esto se pone de manifiesto en que los caudales de base se mantienen muy elevados, reflejando la gran importancia que demuestra la escorrentía subsuperficial en ambientes forestales.

En la parte inferior de la Figura 4 se muestra el periodo seco, comprendido entre los meses de junio y septiembre del mismo año. La precipitación registrada alcanzó los 385,2 mm (un volumen incluso superior al del periodo anterior). Sin embargo, a pesar de la elevada intensidad de algunos eventos (hasta $10 \mathrm{~mm}$ en 5 minutos), hecho típico del periodo estival, apenas se produce respuesta en la cuenca. Durante todo el periodo sólo se generan 3,1 mm de escorrentía ( $0,8 \%$ de la lluvia), siendo el caudal medio durante este periodo de $0,31 \mathrm{~s}^{-1} \mathrm{~km}^{-2}$.

El comportamiento hidrológico del periodo seco, en comparación con el periodo más húmedo, se debe a dos factores fundamentales. En primer lugar, por los más altos valores de interceptación. En estas condiciones alrededor de un $25 \%$ del agua de las precipitaciones queda interceptada en la copa de los árboles (SERRANO-MUELA et al., 2008a). En segundo lugar, los valores de evapotranspiración potencial superan con creces el volumen de precipitación acumulado, lo cual acentúa la situación de déficit hídrico. Esto se pone de manifiesto en la evolución de la altura del nivel freático, cuyas variaciones no son proporcionales a las precipitaciones, en comparación con lo observado durante la estación más húmeda.

La Figura 4 confirma que la respuesta hidrológica en la cuenca de San Salvador sólo se produce en unas condiciones muy determinadas, de manera que las crecidas solamente se producen cuando el nivel freático registrado en el piezómetro de solana alcanza un determinado umbral (aproximadamente $50 \mathrm{~cm}$ por debajo de la superficie). El nivel piezométrico nunca ha alcanzado la superficie en los puntos en que se viene registrando, lo que es indicativo de que el principal mecanismo de generación de escorrentía en las laderas con cubierta forestal es la escorrentía subsuperficial (FREEZE, 1972; WEYMAN, 1973, BEVEN, 1982).

\section{Discusión y conclusiones}

Los datos hidrológicos utilizados en este estudio han confirmado la nolinealidad de la respuesta hidrológica en relación con el volumen de 
precipitación. La serie de datos comprendida entre los años 1999-2006 ha permitido realizar una caracterización de la variabilidad temporal de la respuesta hidrológica en la cuenca de San Salvador.

La respuesta hidrológica en la cuenca de San Salvador está asociada a dos aspectos principalmente, el estado hídrico de la cuenca, representado por el caudal de base al inicio de la crecida y por la altura del nivel freático, y el volumen total de precipitación. Sólo se registra respuesta cuando se cumplen simultáneamente unas determinadas condiciones del estado hidrológico de la cuenca y de la precipitación: (i) eventos pluviométricos con volúmenes relativamente elevados, por encima de $20 \mathrm{~mm}$ y (ii) cuando el caudal de base es superior a $10 \mathrm{l} \mathrm{s}^{-1} \mathrm{~km}^{-2}$. Las respuestas de mayor magnitud y cuyos coeficientes de escorrentía superan el 0,20, se dan siempre que coinciden ambas situaciones.

La relación entre las diferentes variables utilizadas en el trabajo (volumen e intensidad máxima de precipitación y caudal de base) con respecto al coeficiente de escorrentía es semejante a la observada en otros estudios realizados en montaña mediterránea (LANA-RENAULT, 2007; LATRON et al., 2008) o en otras zonas de clima templado húmedo no mediterráneas (JORDAN, 1994). No obstante, los coeficientes de correlación obtenidos en la cuenca de San Salvador son siempre inferiores a los presentados en estos trabajos. De igual forma, las correlaciones obtenidas entre estas mismas variables y el incremento máximo de caudal, para episodios lluviosos superiores a $20 \mathrm{~mm}$, son inferiores a las obtenidas en otros trabajos del ambiente mediterráneo.

La correlación significativa entre el incremento máximo de caudal y el coeficiente de escorrentía, como variables dependientes, con respecto al caudal de base inicial, como variable independiente, para los episodios lluviosos menos voluminosos (inferiores a $20 \mathrm{~mm}$, Tabla 1), indica el gran protagonismo que muestra el estado de la reserva hídrica de la cuenca en la magnitud de su respuesta hidrológica.

Los resultados que refleja la Figura 3 sobre la influencia de la precipitación y el caudal de base en el coeficiente de escorrentía, son parecidos a los ya descritos por LATRON (2003) en la cuenca mediterránea de Can Vila. Sin embargo, para lluvias inferiores a $20 \mathrm{~mm}$ y para un mismo caudal de base, la respuesta hidrológica observada en la cuenca de San Salvador es siempre menor a la observada en Can Vila (donde el bosque cubre sólo el 30\% de la cuenca). Algo similar ocurre en la cuenca forestal de la Rinconada, en el oeste de la Península Ibérica, cuya respuesta es modesta y necesita que la lluvia supere un determinado umbral (alrededor de $30 \mathrm{~mm}$ ) (MARTÍNEZFERNÁNDEZ et al., 2005). 
Al contrario de lo que se ha observado en otras cuencas mediterráneas (CEBALLOS \& SCHNABEL 1998, LANA-RENAULT, 2007; LATRON et al., 2008; NADAL-ROMERO et al., 2008), en San Salvador destaca la escasa importancia que la intensidad de la lluvia tiene sobre la respuesta hidrológica, coincidiendo con las principales conclusiones de HEWLETT et al. (1977). Estos autores demostraron que la respuesta hidrológica en cuencas cubiertas por vegetación dependía muy poco de la intensidad de la lluvia y que el comportamiento hortoniano se limitaba a las áreas con escasa cubierta vegetal.

La especificidad de la respuesta hidrológica en la cuenca de San Salvador puede ser parcialmente explicada por algunas de las características intrínsecas de la cuenca. Por un lado el importante papel de la interceptación de la lluvia por el bosque, dado el importante grado de cobertura del suelo, y por otro, las características de los suelos, propias de un ambiente forestal poco o nada alterado.

La cantidad de agua que no llega al suelo puede ser muy elevada en función de la especie arbórea y de la época del año, con independencia de la intensidad y duración de las precipitaciones (HAMILTON \& ROWE, 1949; ZINKE, 1967; GARCÍA-ORTIZ, 2006). En el caso de climas intermedios o con estaciones muy marcadas, LLORENS (1997) afirma que el bosque juega un papel hidrológico importantísimo a través de la interceptación a pesar de la intensidad de las precipitaciones, frecuentes sobre todo en periodos estivales. Teniendo en cuenta las especies características del área de estudio, puede hacerse una estimación sobre la importancia de la cantidad de agua que no alcanza el suelo:

-Bajo Pinus sylvestris, una de las especies perennifolias que aparece en toda la superficie de la cuenca, la interceptación es muy variable (8-48 \%), según han observado varios autores (ALVERA, 1976 y 1977; SANTA REGINA, 1995; TARAZONA et al. 1996; LLORENS, 1997). Los datos obtenidos en San Salvador durante dos años de estudio, indican valores de interceptación alrededor del 22\% (SERRANO-MUELA et al. 2008a).

-Bajo cubierta de quercinias (Quercus faginea), estudios previos indican que la cantidad de precipitación que no llega al suelo se sitúa entre el 15\% y el 32\% (MOSELLO et al. 2002; MORENO, 1994; MORENO et al., 2001; MARTÍNEZ-FERNÁNDEZ et al., 2005). Para esta especie se obtuvieron valores en el área de estudio de alrededor del 23\% (Serrano-Muela et al., 2008a).

-Finalmente, bajo Fagus sylvatica la interceptación registrada en otras zonas de estudio oscila entre el 14 y el $29 \%$ (DIDON-LESCOT, 1998; MOSELLO et al., 2002). Los valores de interceptación obtenidos en el área de 
trabajo bajo cubierta de haya varían bastante durante el periodo vegetativo activo y el de reposo: los valores de interceptación medios durante el periodo invernal se sitúan en torno al $17 \%$ y en el periodo activo al 28\% (SERRANOMUELA et al., 2008a).

Por otro lado, los suelos forestales tienen generalmente una capacidad de infiltración superior a la de áreas de pasto o con vegetación escasa, debido principalmente a los macroporos que generan las raíces del los árboles (BOSCH \& HEWLETT, 1982) o a una menor degradación del suelo (SEEGER \& FREVEL, 1999; SEEGER, 2001). En la cuenca de San Salvador, los suelos alcanzan espesores de más de un metro, con una elevada presencia de raíces en los horizontes superiores. Al mismo tiempo presentan, sobre todo en la vertiente umbría, una capa orgánica hidráulicamente efectiva (SEEGER \& BEGUERÍA, 2003) que favorece la infiltración.

Estas características de los suelos sugieren que, durante los meses en los que el balance entre precipitación y ETP es negativo, el agua que alcanza el suelo se infiltra rápidamente. Por ello la capacidad de generación de escorrentía es muy reducida, pues el déficit hídrico que presenta el suelo puede llegar a ser muy superior al volumen registrado en cualquier tormenta de verano. Únicamente a medida que se producen las precipitaciones durante la estación más húmeda, coincidiendo con una menor temperatura media y, especialmente, cuando las plantas han detenido su actividad vegetativa, es cuando los acuíferos comienzan a recargarse y se dan unas condiciones favorables para la generación de crecidas.

La observación del nivel piezométrico (Figura 4) ha resultado esencial en esta cuenca para explicar la respuesta hidrológica. Como ya apuntaron HURSH \& BRATER (1941), CAPPUS (1960), HEWLETT \& HIBBERT (1967), SKLASH \& FARVOLDEN (1979) o PRICE (1997), entre otros, las fluctuaciones producidas por los niveles piezométricos condicionan la aparición de procesos subsuperficiales y de saturación del suelo, contribuyendo de manera muy significativa a la respuesta del caudal.

El comportamiento del nivel freático (Figura 4) refleja una marcada estacionalidad en la dinámica de las reservas hídricas de la cuenca. En los piezómetros instalados el nivel freático nunca ha alcanzado la superficie, y esto sugiere que en la mayoría de la cuenca el suelo no alcanza nunca la saturación, excepto en áreas muy puntuales (pistas forestales o áreas desnudas). La elevada capacidad de infiltración de los suelos favorece que la escorrentía predominante sea la subsuperficial, al contrario de lo que ocurre en otras cuencas mediterráneas (PETERS et al., 2003; LATRON, 2003; LANARENAULT, 2007) donde si se dan procesos de saturación. Además, las fuertes pendientes que dominan en la cuenca de San Salvador facilitan la rápida 
circulación subsuperficial del agua, de manera que tras la lluvia el descenso del nivel freático se produce con cierta rapidez.

La interceptación de la lluvia por el bosque y las propiedades de los suelos confieren a la cuenca de San Salvador los rasgos más relevantes de su comportamiento hidrológico. Uno de ellos es la total ausencia de respuesta hidrológica en aquellos momentos del año en que las condiciones hidrológicas no son tan favorables (periodo de precipitaciones escasas con altos valores de evapotranspiración y de interceptación). Este hecho llama la atención cuando se compara la respuesta con otros ambientes próximos y cuyo uso del suelo es distinto (GARCÍA-RUIZ et al., 2005 y 2008; LANARENAULT et al., 2007 y 2009).

Durante el año hidrológico 2005-2006, en la cuenca forestal de San Salvador sólo se registraron 6 eventos de crecida, mientras en la vecina cuenca de Arnás (donde predomina la vegetación arbustiva sobre antiguos campos de cultivo abandonados) se registraron 12 eventos significativos. Sin embargo, ambos casos contrastan notablemente con las 44 crecidas registradas en la cuenca de Araguás, que se encuentra a muy pocos kilómetros de las anteriores pero se diferencia por su elevada degradación, con casi un tercio de su superficie ocupado por cárcavas. Esto remarca la importancia de la cubierta vegetal en la frecuencia y magnitud de los picos de crecida (BEGUERÍA et al., 2003; LÓPEZ-MORENO et al., 2006) pues sin tener en cuenta siquiera las condiciones hidrológicas antecedentes, las cuencas forestales registran volúmenes de caudal inferiores y picos de crecida más moderados (BONELL, 1993).

\section{Agradecimientos}

Este trabajo ha sido realizado con el apoyo de los siguientes proyectos: "Procesos y balances de sedimentos a diferentes escalas espaciales en ambientes mediterráneos: Efectos de las fluctuaciones climáticas y los cambios de uso del suelo" (PROBASE, CGL2006-11619/HID) y "Comportamiento y modelización espacio temporal de la transferencia de sedimento en distintos usos del suelo" (CETSUS, CLG2007-66644-C04-01), ambos financiados por la CICYT (Ministerio de Educación y Ciencia), y ACQWA (FP7-ENV-2007-1), financiado por la Comisión Europea, y “Desarrollo y validación de modelos acoplados hidrológicos e hidráulicos en cuencas a diferentes escalas espaciales" (PI032/08), financiado por la Diputación General de Aragón (DGA). La monitorización de la cuenca también ha sido financiada mediante el proyecto RESEL (CSIC y Ministerio de Medio Ambiente). Asimismo, Pili Serrano-Muela cuenta con una beca 
predoctoral I3P concedida por el CSIC en el contexto del Programa Social Europeo y Estela Nadal-Romero cuenta con un contrato postdoctoral del Ministerio de Ciencia e Innovación, mediante el Programa Nacional de Movilidad de Recursos Humanos del Plan nacional de I-D+I 2008-2011. Finalmente, los autores agradecen su ayuda a Noemí Lana-Renault, Carlos Martí Bono, Jérôme Latron y Sergio Valdivielso.

\section{Referencias}

ALVERA, B., 1976. Contribución al estudio de la interceptación de las precipitaciones atmosféricas en el pinar de San Juan de la Peña. Publicaciones Centro Pirenaico Biología Experimental, 7 (1): 95-100.

ALVERA, B., 1977. Interceptación de las precipitaciones atmosféricas en un pinar altoaragonés: $1^{\text {er }}$ año de observaciones (1971-72). Publicaciones Centro Pirenaico Biología Experimental, 8: 7-13.

ANDRÉASSIAN, V., 2004. Water and forest: from historical controversy to scientific debate. Journal of Hydrology, 291: 1-27.

BEVEN, K., 1982. On subsurface stormflow: an analysis of response times. Hydrological Sciences Journal, 27 (4): 505-521.

BEGUERÍA, S., LOPEZ-MORENO, JI., LORENTE, A., SEEGER, M., \& GARCÍA-RUIZ, JM., 2003. Assesing the Effect of Climate Oscillations and land-Use Changes on Streamflow in the Central Spanish Pyrenees. Ambio, 32: 283-286.

BONELL, M., 1993. Progress in the understanding of runoff generation dynamics in forests. Journal of Hydrology, 150: 217-275.

BOSCH, J.M. \& HEWLETT, J.A., 1982. A review of catchment experiments to determine the effect of vegetation changes on water yield and evapotranspiration. Journal of Hydrology, 55: 3-23.

CAPPUS, P., 1960. Bassin expérimental d'Alrance. Étude des lois de l'écoulement. Application au calcul et à la prévision des débits. La Houille Blanche, A: 493-520.

CEBALLOS A.. \& SCHNABEL. S., 1998. El ciclo del agua en una dehesa extremeña: resultados de ocho años de estudio en la cuenca del Guadalperalón. Quercus, 145: 31-34.

COSANDEY, C., 1986. De l'origine de l'écoulement rapide de crue, dans un petit basin-versant forestier Breton. Zeitschrift für Geomorphologie, N. F., suppl. Bd. 60: 177-186.

COSANDEY, C., ANDREASSIAN, V., MARTIN, C., DIDON-LESCOT, J.F., LAVABRE, J., FOLTON, N., MATHYS, N. \& RICHARD, D., 2005. The hydrological impact of the mediterranean forest: a review of French research. Journal of Hydrology, 301: 235-249. 
CREUS, J., 1983. El clima del Alto Aragón Occidental. Monografías del Instituto de Estudios Pirenaicos, 109 pp. Jaca (Huesca).

DIDON-LESCOT, J.F., 1998. The importance of throughfall in evaluating hydrological and biogeochemical fluxes: example of a catchment (MontLozère, France). In: Conference on Catchment Hydrological and Biochemical Processes in Changing Environment, Liblice (Chec Republic), 17-20.

FREEZE, R.A., 1972. Role of subsurface flow in generating surface runoff 2. Upstream source areas. Water Resources Research, 8 (5): 1272-1283.

GARCÍA-ORTIZ, E., 2006. Efecto de la estructura de la copa en la partición de lluvia de tres especies arbustivas en clima semiárido. Tesis Doctoral inédita, Universidad de Almería. 404 pp.

GARCÍA-RUIZ, J.M., ARNÁEZ, J., WHITE, S.M., LORENTE, A. \& BEGUERÍA, S., 2000: Uncertainty assessment in the prediction of extreme rainfall events: an example from the Central Spanish Pyrenees. Hydrological Processes, 14: 887-898.

GARCÍA-RUIZ, J.M., ARNÁEZ, J., BEGUERÍA, S., SEEGER, M., MARTÍ, C., REGÜÉS, D., LANA-RENAULT, N. \& WHITE, S., 2005. Flood generation in an intensively disturbed, abandoned farmland catchment, Central Spanish Pyrenees. Catena, 59: 79-92.

GARCÍA-RUIZ, J.M., REGÜÉS, D., ALVERA, B., LANA-RENAULT, N., SERRANO-MUELA, P., NADAL-ROMERO, E., NAVAS, A., LATRON, J., MARTÍ-BONO, C. \& ARNÁEZ, J., 2008. Flood generation and sediment transport in experimental catchments affected by land use changes in the central Pyrenees. Journal of Hydrology, 356 (1-2): 245-260.

GUOJING Y., DUNING X., LIHUA Z. \& CUIWEN T., 2005. Hydrological effects of forest landscape patterns on the Qilian mountains. A case study of two catchments in Northwest China. Mountain Research and Development, 25: 262-268.

HAMILTON, E.L. \& ROWE, P.B., 1949. Rainfall interception by chaparral in California. Calif.Forestry and Range Expt. Sta. in coop. with Calif. Div. of Forestry. $43 \mathrm{pp}$.

HEWLETT, H. \& HIBBERT, A.R., 1967. A Review of Catchment Experiments to Determine the Effect of Vegetation Changes on Water Yield and EvapoTranspiration. Journal of Hydrology, 55 (1-4): 3-23.

HEWLETT, J.D., FORTSON, J.C. \& CUNNINGHAM, G.B., 1977. Effect of rainfall intensity on storm flow and peak discharge from forest land. Water Resources Research, 13(2): 259-266.

HIBBERT, A.R., 1967. Forest Treatment effects on water yield, Reprint from Proceedings of International Symposium on Forest Hydrology, Penn State University, 1965, Pergamon Press, New York, 527-543. 
HURSH, C.R. \& BRATER, E.F., 1941. Separating storm-hydrographs from small drainage-areas into surface- and subsurface-flow. Transactions of the American Geophysical Union, 22: 863-871.

LANA-RENAULT, N., 2007. Respuesta hidrológica y sedimentológica en una cuenca de montaña media afectada por cambios de cubierta vegetal: la cuenca experimental de Arnás, Pirineo Central. Tesis Doctoral inédita, Universidad de Zaragoza, Zaragoza, $314 \mathrm{p}$.

LANA-RENAULT N., REGÜÉS D., MARTÍ-BONO C., LATRON, J., ARNÁEZ, J., NADAL, E., SERRANO, P. \& GARCÍA-RUIZ J.M., 2007. Temporal variability in the relationships between precipitation, discharge and suspended sediment concentration in an experimental mountain catchment. Nordic Hydrology, 38 (2): 139-150.

LANA-RENAULT, N., NADAL-ROMERO, E., SERRANO-MUELA, P., LATRON, J., REGÜÉS, D., MARTÍ-BONO, C. \& GARCÍA-RUIZ, J.M., 2009. Hydrological dynamics and sediment response in three research basins with different land cover. In: A. Herrmann and S. Schumann (Eds.), International Workshop on Status and Perspectives of Hydrology in Small Basins. Landschaftsökologie und Umweltforschung, Heft 50. T.U. Braunschweig, Braunschweig: 121-125.

LATRON, J., 2003. Estudio del funcionamiento hidrológico de una cuenca mediterránea de montaña (Vallcebre, Pirineos Catalanes). Depto. de Geoquímica, Petrología i Prospecció Geológica, Facultad de Geología. Barcelona, Universitat de Barcelona, 269 pp.

LATRON, J., SOLER, M., LLORENS, P. \& GALLART, F., 2008. Spatial and temporal variability of the hydrological response of a small Mediterranean basin (Vallcebre, Eastern Pyrenees) Hydrological Processes, 22 (6): 775-787.

LATRON, J. \& GALLART, F. 2008. Runoff generation processes in a small Mediterranean research catchment (Vallcebre, Eastern Pyrenees). Journal of Hydrology, 358: 206-220.

LÓPEZ-MORENO, J.I., BEGUERÍA, S. \& GARCÍA-RUIZ, J.M., 2006. Trends in high flows in the Central Spanish Pyrenees: response to climatic factors or to land use change? Hydrological Sciences Journal, 51: 1039-1050.

LÓPEZ-MORENO, J.I., GARCÍA-RUIZ, J.M. \& BENISTON, M., 2008. Environmental Change and water management in the Pyrenees. Facts and future perspectives for Mediterranean mountains. Global and Planetary Change, 66 (3-4): 300-312.

LLORENS, P., POCH, R. LATRON, J. \& GALLART, F., 1997. Rainfall interception by a Pinus sylvestris forest patch overgrown in a Mediterranean mountainous abandoned area. I. Monitoring design and results down to the event scale. Journal of Hydrology, 199: 331-345. 
LULL, H.W. \& REINHART K.G., 1967. Increasing Water Yield in the Northeast by Management of Forested Watersheds USDA Forest Service Research Paper NE-66, Northeastern Forest Experiment Station, Upper Darby, PA, 45 pp.

JORDAN, J.P., 1994. Spatial and temporal variability of stormflow generation processes on a Swiss catchment. Journal of Hydrology, 153: 357-382.

MARTÍNEZ-FERNÁNDEZ, J., CEBALLOS BARBANCHO, A., HERNÁNDEZ SANTANA, V., CASADO LEDESMA, S. \& MORÁN TEJEDA, C., 2005. Procesos hidrológicos en una cuenca forestal del Sistema Central: cuenca experimental de Rinconada. Cuadernos de Investigación Hidrológica, 31: 7-25.

MONTSERRAT RECODER, P., 1971. El ambiente vegetal jacetano. Pirineos, 101: 5-22.

MORENO, G., 1994. Balances de agua y nutrientes en rebollares (Quercus pyrenaica Willd.) de la vertiente salmantina de la Sierra de Gata. Ph.D. Thesis, Universidad de Salamanca, Salamanca, $470 \mathrm{pp}$.

MORENO, G., GALLARDO, J.F. \& BUSSOTTI, F., 2001. Canopy modification of atmospheric deposition in oligotrophic Quercus pyrenaica forests of an unpolluted region (central-western Spain). Forest Ecology and Management, 149 (1-3): 47-60.

MOSELLO, R., BRIZZIO, M.C., KOTZIAS, D., MARCHETTO, A., REMBGES, D. \& TARTARI, G., 2002. The chemistry of atmospheric deposition in Italy in the framework of the National Programme for Forest Ecosystems Control (CONECOFOR). Journal of Limnology, 61 (1): 77-92.

NADAL-ROMERO, E., 2008. Las áreas de cárcavas (badlands) como fuente de sedimento en cuencas de montaña: procesos de erosión, meteorización, erosión y transporte en margas del Pirineo Central. Tesis Doctoral inédita. Universidad de Zaragoza, $434 \mathrm{pp}$.

NADAL-ROMERO, E. LATRON, J., LANA-RENAULT, N., SERRANOMUELA, P., MARTÍ-BONO, C. \& REGÜÉS. D., 2008. Temporal variability in hydrological response within a small catchment with badland areas, central Pyrenees. Hydrological Sciences, Journal des Sciences Hydrologiques, 53(3): 629-639.

PETERS, E., FREER, J. \& AULENBACH, B.T., 2003. Hydrological dynamics of the Panola Mountain Research Watershed, Georgia. Ground Water, 41(7): 973-988.

PUIGDEFÁBREGAS J. \& CREUS, J., 1976. Pautas espaciales de variación climática en el Alto Aragón. Publicaciones del Centro Pirenaico de Biología Experimental, 7 (1): 23-34.

PRICE, J., 1997. Soil moisture, water tension, and water table relationships in a managed cutover bog. Journal of Hydrology, 202(1-4): 21-32. 
SANTA REGINA, I. 1995. Ciclos biogeoquímicos en bosques de la Sierra de Béjar (Salamanca). Retorno de bioelementos por medio del agua de lluvia. Pirineos, 145-146: 65-80.

SEEGER, M., 2001. Boden und Bodenwasserhaushalt als Indikatoren der Landdergradierung auf extensivierten Nutzflächen in Aragón/Spanien. Dissertation, Institut für Physische Geographie, Freiburg i Br., Freiburger Geographische Hefte, 63, 184 pp.

SEEGER, M. \& FREVEL, C., 1999. Factores hídricos y erosionabilidad en el gradiente edafoclimático Depresión del Ebro / Pirineo Central. Cuadernos de Investigación Geográfica, 25: 85-110.

SEEGER, M. \& BEGUERÍA, S., 2003. La respuesta hidrológica en dos cuencas experimentales con diferentes usos del suelo en el Pirineo aragonés. En: El cambio en el uso del suelo y la degradación del territorio en España. Marzolff, Ries, de la Riva, Seeger (Eds), 203-221.

SERRANO-MUELA, P., REGÜÉS, D., LANA-RENAULT, N. \& NADALROMERO, E., 2008a. Estudio de la trascolación bajo diferentes tipos de cubierta forestal durante el periodo fenológico con hojas en el Pirineo Central Español. En: / Trabajos de Geomorfología en España, 2006-2008/ (J. Benavente y Gracia, F.J.). Sociedad Española de Geomorfología, Cádiz, 101-104.

SERRANO-MUELA, M.P., LANA-RENAULT, N., NADAL-ROMERO, E., REGÜÉS, D., LATRON, J., MARTÍ-BONO, C. \& GARCÍA-RUIZ, J.M., 2008b. Forests and their hydrological effects in mediterranean mountains: the case of the central Spanish Pyrenees. Mountain Research Development, 28. (3-4): 279-285.

SKLASH, M.G., FARVOLDEN, R.N., 1979. The role of groundwater in storm runoff. Journal of Hydrology, 43: 45-65.

STEDNICK, J.D., 1996. Monitoring the effects of timber harvest on annual water yield. Journal of Hydrology, 176 (1-4): 79-95.

TARAZONA, T., SANTA REGINA, I. \& CALVO, R., 1996. Interception, throughfall and stemflow in two forest of the "Sierra de la Demanda" in the province of Burgos. Pirineos, 147-148: 27-40.

TRIMBLE S.W., WEIRICH, F.H. \& HOAG, B.L., 1987. Reforestation and the reduction of water yield on the southern piedmont since circa 1940. Water Resources Research, 23 (3): 425-437.

WEYMAN, D.R., 1973. Measurements of the downslope of water in a soil. Journal of Hydrology. 20: 267-288.

WHITEHEAD, P.G. \& ROBINSON, M., 1993. Experimental basin studies-an international and historical perspective of forest impacts. Journal of Hydrology, 145: 217-230. 
LA VARIABILIDAD TEMPORAL DE LA RESPUESTA HIDROLÓGICA EN UNA PEQUEÑA...

ZABALETA, A., 2007. Análisis de la respuesta hidro-sedimentaria en pequeñas cuencas de Gipúzkoa. Tesis Doctoral. Universidad del País Vasco, 347 pp.

ZINKE, P. J., 1967. Forest interception studies in the Unites States. En Shopper, W. and Lull, H. (Eds.): Forest Hydrology. N.York, 137-161. 